\title{
ANCIENT ROME WORLDWIDE LINKS: SHARING KNOWLEDGE TO PRESERVE THE ROOTS
}

\author{
P. Paolini ${ }^{a} *$, F. Allegrini Simonetti ${ }^{\text {b }}$, G. Forti $^{\text {c }}$, A.Corrao $^{d}$ \\ ${ }^{a}$ Dept. of History, Representation, and Restoration of Architecture (D.S.D.R.A), "SAPIENZA" University, \\ 00100 Rome, Italy - priscilla.paolini@uniroma1.it \\ ${ }^{\mathrm{b}}$ Collectivité territoriale de Corse, Direction de la culture et Patrimoines, Service des patrimoines, secteur archéologie, \\ 20187 Ajaccio, archaeologist - fallegrini-simonetti@ct-corse.fr \\ ${ }^{c}$ ESRI Italia, Rome, Italy-geologist, GIS expert - gforti@esriitalia.it \\ ${ }^{\mathrm{d}}$ Mi.B.A.C. (Ministero per i Beni e le Attività Culturali), Rome, Italy- photographer, Cultural Heritage documentation \\ - alfredo.corrao@beniculturali.it
}

KEY WORDS: Cooperation, Research, Cultural heritage, Archaeology, Architecture, GIS, Photography, LIDAR.

\begin{abstract}
:
Following the collaboration agreement between the SAPIENZA, S.D.R.A. Department and the "Collectivité territoriale de Corse, secteur Archéologie", this project tried to set, accomplished on the archaeological site of the ancient roman city of Aléria, a complex program of selected dataset structured for many different uses and fruitions.

As for any kind of survey, the initial project definition, described in this paper, constitutes the most delicate part of the work, in this instance a certain additional significance it has to be given to it, cause of the multiple interests focalized on the Aléria site, where a new digging season is expected after a sixty years long interruption.

The process can be synthesized as follows: various surveying technologies were applied on the site, as 3D Laser scanning, Topography, and GPS; Dense Stereo Matching was accomplished on a sample object there excavated and actually exposed in the local Carcopino Museum, while Computational Photography techniques were realized on an object exposed in Rome in the Etruscan Museum of "Villa Giulia" as the other twin found and exposed in Aléria, to be a purpose for future collaborations. A GIS and WEBGIS workflow followed, using a specific application in its latest version, thus collecting all of the actual and previous documents, providing to build up a complete 3D geo-database with a space and time referenced 3D Web scene to share in the GIS online Cloud Platform. These applied procedures aim to spread the complex results, articulated in different sets on the social media world.
\end{abstract}

\section{INTRODUCTION}

The main theme of this research was defined during a cultural visit in the Middle East coast of Corsica, focused on the traces of the Roman Empire not so far from its core. Therefore the ideas exchange between the authors of this paper, as specialists in different professional fields, brought to first evidence the total fault of detailed surveys of the area, where the archaeological excavations, lasted four decades, from 1920-22 till around 1960-61, and leaded on the monumental center of the roman urbs by monsieur Jean Jehasse as the research director, resulted into a general-plan drawing and in a very synthesized report, both published in 1963. From this critical fault we decided to project a multi-resolution set of 3D survey models focused either on the archeological site either on some roman and pre-roman objects earlier retrieved, to make it part of the total excavation data and contents resumption, all correlated in a four-dimensional WEB Geo-database. Thus intending to constitute the new start point for the site renaissance, while facing the reality of long time interrupted excavations, waiting for the beginning of the new digging season to be in a couple of years.

After more or less twenty years' worldwide experience in 3D laser scan surveying (Lidar), the geo-referenced point cloud model and its textured surface has been accepted as a scientifically affordable digital system to document, even territories or Cultural Heritage artifacts, much younger and in consolidation even if part of stereo-photogrammetry family is the Dense Stereo Matching (DSM) technique, as an alternative on creating a 3D point cloud model, RGB textured, scaled and geo-referenced. Based on the geometric theory of forward intersection in space, it varies the original method of stereo restitution in recognizing homologous points by the use of multiple image sets, thus extra views succeed on reducing ambiguity in matching, their wider range creates fewer "holes", and have better noise properties and an increased depth precision. Hence, differently created point cloud models can be combined such improving the quality and descriptive completeness of the final multi-resolution $3 \mathrm{D}$ model. To reach a more qualitative result, both models are linked together by the use of targets, at most automatically recognized in both type of software and then measured with total stations and GPS for global geo-referencing and easy GIS and WEB import.

An additional value to this project is provided by the use of Computational Photography as a valid instrument to promote digital documentation and study of Cultural Heritage. A part of this world is represented by the Giga Pixel Image, this multiimage stitching technique, is here applied on an object, known as the Capena "plate with the elephant", exposed in the Villa Giulia National Etruscan museum of Rome, the first of a pair where the second is in the Carcopino museum of Aléria, on which our final plan is to realize the coupled Giga pixel Image. By loading both Giga images in dedicated viewers it will be possible to realize their direct comparison, a side by side or

\footnotetext{
* Corresponding author.
} 
layered evaluation, a metric measurement in real time, as an overlaying drawing and its relative annotations. All of this work can be attached as a link to the Web GIS Cloud project, hosted inside the tridimensional model of the Carcopino museum.

Moreover, the very last evolution of dedicated GIS software, resolving the serious limit caused by the big size of 3D survey models, make now possible to import millions of point cloud models as much as small detailed ones, including space coordinates (x, y, z), RGB and intensity data, all together into a Geographical Information System, where all the old and new contents about the archaeological site are going to be finally collected. The even new opportunity of realizing an accurate 3D reconstruction of the geo-referenced site and its related surroundings, making all directly disposable on the WEB, has become one of the goals of this pilot-plan of research.

\section{ALERIA AND THE REAPPROPRIATION OF DATA BY THE DIGITALISATION OF THE GLOBAL AND THE PARTICULAR ${ }^{\text {b }}$}

\section{2-1 State of Knowledge: the Territory}

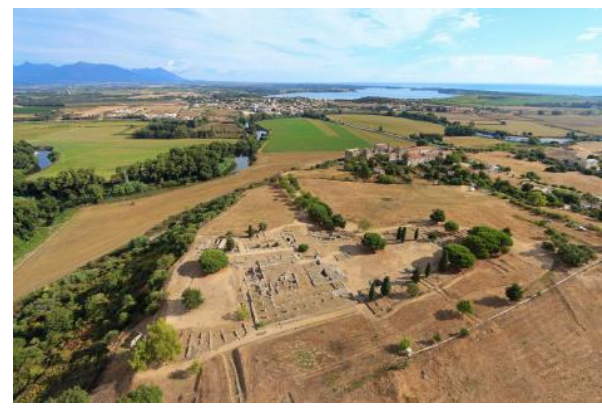

Figure 1. City of Aleria () S. Aude balloide Photo - CTC 2012.

Located near the hamlet of $U$ Forte, The ancient city of Aléria is set on a remote tabular hill $3 \mathrm{~km}$ far from the sea (Figure 1.) The city center covers 6 acres which is the geographical epicenter of other ancient sites such as the Roman baths of Santa Laurina $(400 \mathrm{~m})$ and the pre-Roman necropolis of Casabianda $(800 \mathrm{~m})$ (Cesari, 2010). The location has a diverse landscape. This area, characterized by cultivated plains, wetlands, steep hills, Mediterranean bush and a large coastline, is unique in Corsica by the convergence of all these assets.

\subsection{History}

\section{Recent Prehistory}

In the $5^{\text {th }}$ century BC Alalie name appears in a text of the Greek historian Herodotus. He mentions the arrival of a group of Phoenicians driven away of Asia Minor by the Persians in 545 BC. However, no architectural remains of the Greek settlement suggested by these texts have ever been recovered.

\section{Etruria and Rome}

The $5^{\text {th }}$ and $4^{\text {th }}$ centuries before the Christian era are well documented by the excavation of the pre-Roman necropolis of Casabianda. The Etruscan character of this site and associated funerary objects infers the existence of a strong relationship, whose nature remains to be clarified, between Aléria and northern Etruria.

If some traces of Roman influence appear in the course of the $2^{\text {nd }}$ century $\mathrm{BC}$, it is only from the $1^{\text {st }}$ century that texts inform us about the Roman city erected over the first century BC after colonization has been subsequently ordered by Sylla in 81 BC, by Caesar in $46 \mathrm{BC}$ and Octave in $32 \mathrm{BC}$. The city prospers throughout the Early Empire as a military port and emporium.

\subsection{First Excavations}

The remains uncovered, the forum, the amphitheater and the ramparts, correspond respectively to the center and the south end of the Roman city. The excavations that have unearthed these areas were undertaken in 1955 by archaeologists Jean and Laurence Jehasse (Jehasse, 1997). After clearing these areas, they devoted themselves in the early 60 s searching for the preRoman necropolis, returning only sporadically to work on the Roman city during the following decades.

\subsection{The Site}

The forum area: The main buildings and monuments are built around a forum flanked by two covered porches that protect the facades of the tabernae. Two temples are facing on both sides of the forum and two large houses with a peristylium are present at the east and west ends. The thermal pools, private or public, are all located to the west, near the highest part of the city.

The amphitheater quarter: $200 \mathrm{~m}$ south of the forum, the amphitheater quarter marks the southern end of the city of Aleria. This sector is characterized by the presence of two successive walls and an amphitheater corresponding to three different timelines.

The pre-Roman rampart ( $4^{\text {th }}$ century $\left.\mathrm{BC}\right)$ : made of limestone blocks surmounted by mud bricks to increase its height (Fig 2) The amphitheater $\left(2^{\text {nd }} 1^{\text {st }}\right.$ century $\left.\mathrm{BC}\right)$ : the cylindrical building masonries, measure $35 \mathrm{~m}$ at its widest point. The Roman Walls $\left(1^{\text {st }} \mathrm{BC}\right)$ : only a short section of the wall, made of concrete, is still visible.

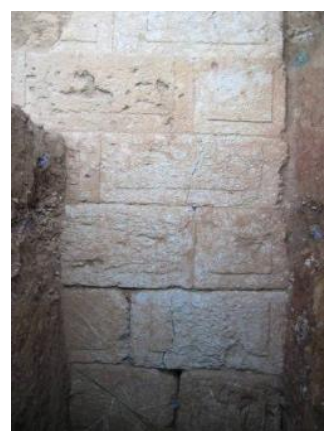

Figure 2. Part of Pre-Roman rampart@ F F. Allegrini-Simonetti CTC 2011

\subsection{Conservation, Research and Development}

A program aiming to reassess the scientific data is currently under way at Aléria; this is linked to the three fundamentals of site management: conservation, research and development.

Various projects led by specialists, including a complete restudy of the remains (Coutelas et al., 2012), are laying the foundations for a new beginning for site conservation and for the improvement of public access.

A future campaign of 3D laser scanner and photogrammetric surveys will significantly contribute to the reinterpretation of the site, also supporting the development of the actual masonry conservation program. The 3D surveying techniques will be applied in the next phase for the research new start and the new site excavations, and the results of related studies diffused to the public. 


\section{THE SURVEY MODELS WORKFLOW}

\subsection{D laser scanning, Topography and GPS}

"The excavation implies not only the dismantling but even the reassembling operation, and that is the creation of a story in the tridimensional space... to make destruction useful to a correct reconstruction it's necessary to apply the art of stratigraphy." (A. Carandini). The excavation process of modern Archaeology, to restitute the maximum of information, follows the strict rules of stratigraphy to state the sequence of actions and natural and human activities layered in a specific time and space boundary, singularly distinguished and related to each other. This implies the analytical documentation of any Stratigraphic Unit (S.U.) and the following rearrange of data and the ideal reconstruction. The excavating act proceeds in depth (horizontal or vertical) and when the new layer is determined the previous is permanently destroyed, this implies the performing of a severe documentation process either direct or indirect. This consideration highlights the necessity for all the collected data to have a precise constant reference as in time even in space. About the space location, a solution for any excavation in act is to keep the same geo-references along the site, granting to all Units to be related each other and geo-referenced to the same first origin. As a result the materialization of the network of fixed slots to allow the interchangeable positioning of different targets as topographic and laser scan as GPS antennas, distributed along the site of Aléria, requested the assembly of specific parts, to work as interchangeable hosts (project and components by Instrumetrix, targets by Leica-Geosystems). Moreover, the opportunity of turning around the site without space obstacles, easily performing all the laser scanning and topographic activities, allowed the use of a Phase-based laser scanner.

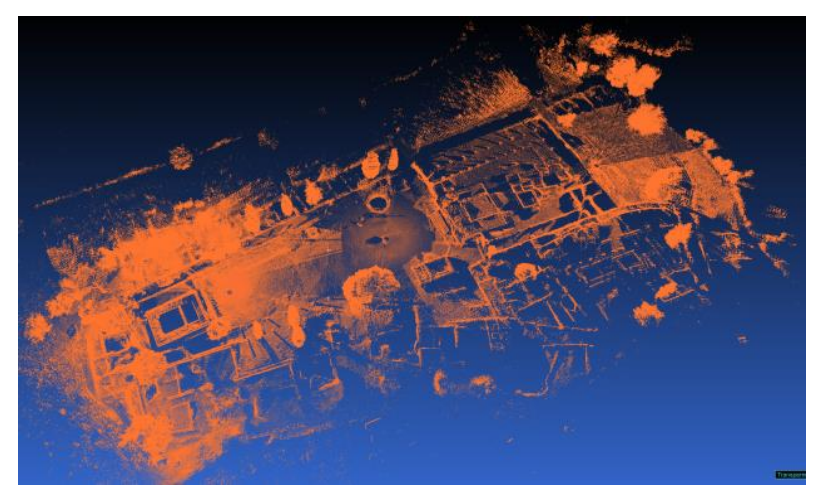

Figure 3. The geo-referenced final model (Cyclone 8.0.2)

The Leica HDS 6000 (laser class 3R) reaching a max. range of $79 \mathrm{~m}$ is better suited to get less noisy point cloud models (noise precision: $2 \mathrm{~mm}$ at $25 \mathrm{~m}, 4 \mathrm{~mm}$ at $50 \mathrm{~m}$ at $90 \%$ albedo) at high scan density $(1.6 \times 1.6 \mathrm{~mm}$ at $10 \mathrm{~m}, 7.9 \times 7.9$ at $50 \mathrm{~m})$ and high speed (500.000 points/sec) with good accuracy in single measurement distance $(4 \mathrm{~mm}$ at $25 \mathrm{~m}, 5 \mathrm{~mm}$ at $50 \mathrm{~m})$. In Archeology accuracy and speed are more valuable properties than range; in addition the internal dual axis sensor (resolution 3.6") constantly switched on leveled each scan model making the registration process faster and easier.

The obtained point cloud, where the medium distance of the surveyed surface was at $15 \mathrm{~m}$ and maximum $30 \mathrm{~m}$ from the scan station, constitutes a sufficiently accurate base for our project. The number of 10 scan' stations, two of which realized to survey an underground water holding Tank, were enough to create in a couple of days the point cloud model more than the topographic survey of targets used as a geo-reference then to be exported in PTS and in LAS format. Lately three GPS points were measured to relate the survey with Lambert 93 oriented coordinates; one of the three stations corresponds to the place where the Jupiter Ammon marble bust was retrieved, to this reference will be then related the $3 \mathrm{D}$ model obtained by dense stereo matching technique applied on the sculpture hosted in the nearby Museum.

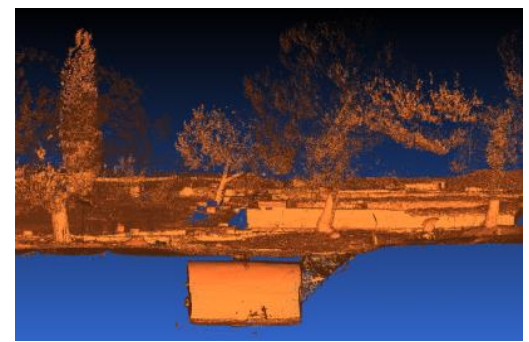

Figure 4. The underground water holding tank Mesh surface

\subsection{Dense Stereo Matching Photogrammetry: The Jupiter Ammon 3D model}

The strong relationship between Archaeology and Photography is based on the common goal to realize a conjunction, to connect traces of the past with present. A landscape image where things are arranged in place creates the association between place and event, such indicating what is important. Thus the relevance of representing how things are displaced in the moment of their retrieval is one of the basics of documentation in Archaeology; even the new digital cameras help this necessity by providing immediately usable results, as typical documents in archaeology are pictures of objects with a measure reference aside or with a measuring frame around. Digital stereo-photogrammetry systems by providing a 3D model allow verifying and measuring an artifact in its tridimensional reality, moreover the opportunity of using partially automated software, adding speed on results, makes it part of the modern spoken language of many archaeologists. Here we consider using these models, not only by enclosing it into Web-GIS for professional use but then again, by creating specific formats, to be relied into the Cloud for public use as Cultural Commons. In our project, a geo-referenced link helps positioning the $3 \mathrm{D}$ object model into its original retrieved location with all the related documents and drawings as geodatabase to support scientists' work, while an additional weblink to the actual localization of the piece in the museum display cabinet acts as a vehicle for educational finalities and for tourism.

The second century bust of Jupiter Ammon, excavated around 1977 in the south part of the site nearby the amphitheater walls and actually exposed in the Carcopino Museum in Aléria, was the sample object of our project.

The Carrara marble sculpture represents Jupiter with a pair of Aries horns encircling his head, a curly beard and two Aries legs on his shoulders; it measures $18.3 \mathrm{~cm}$ in height and $11.3 \mathrm{~cm}$ in width at chest, $10.5 \mathrm{~cm}$ in width at head, $6.7 \mathrm{~cm}$ depth at chest, and $7.3 \mathrm{~cm}$ depth at beard.

The bust was placed for the shooting set on a targeted cube and both over a graded rotary table with a black opaque background. The lightning apparatus was mounted on a stand two meters far, provided with a double mirror reflector and with four bulbs of continuous fluorescent light so as to obtain a wide and uniform diffusion. The color temperature of these lamps is $5400^{\circ} \mathrm{K}$, the same of day-light, for the best color rendering; furthermore offering the great advantage of low energy 
consumption and, mostly, low temperature so to preserve the subject. The utilized camera is a Canon Eos 5D Mark III with a EF $24 \mathrm{~mm}$ f/1.4L II lens, CMOS sensor resolution is 22.3 megapixels (5760x3840), full frame. The camera mounted on a Manfrotto tripod (double bubbled), turned into vertical, was shooting at $19.77 \mathrm{~cm}$ medium distance from the object, ground resolution of 45.7283 micron. The first set of 79 pictures (RAW format) was realized regularly, as suggested, turning around the bust to a fixed $90^{\circ}$ zenithal angle, aperture f/8, ISO 200, shutter $1 / 10$ sec., at fluorescent light, following, one more set of 25 shots was realized without tripod, freely turning around the object, focused on the missing part on top of the head, at natural sunlight, to verify differences in the final result.

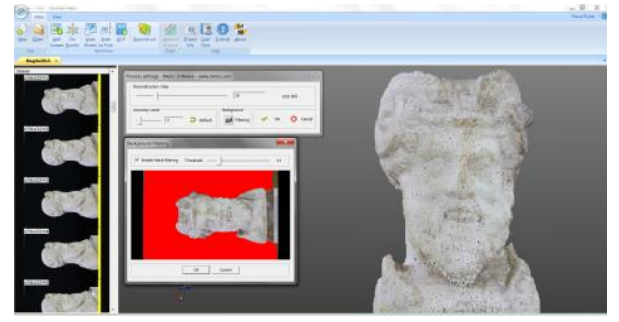

Figure 5. Menci-Umap-Masking and point cloud model

The DSM procedure of the first set was realized either in Umap software (-http://www.menci.com/evo-close-range/11-evo/3umap- educational release) using pre-calibrated camera data or in PhotoScan Professional (PSPro - 0.9.1 http: // Agisoft. ru / products / photoscan / professional - evaluation release) without pre-calibration as a test (even if free software "Lens" is provided for pre-calibration). Both software worked very well on the data elaboration, even though using different mask tools to exclude the dark uniform background (even if, in an on field set, a discontinuous background shouldn't give problems to modeling, actually the use of masks, to exclude any unwanted background, is recommended ). While PSP provides" intelligent scissors" tool to work on image one by one, Umap, by a tool specifically set for our project uses(uMap_64_2.0.0 2013-0620) a color channel filter that, set only on a single image, automatically applies to the whole, incredibly speeding up the procedure(FIG 5). We can't say the same about the attempt of elaborating the other uncontrolled and not regularly overlapped set of 25 images (FIG6), there the only solving procedure was to match the new set in Photo Scan Pro to the previously realized model.

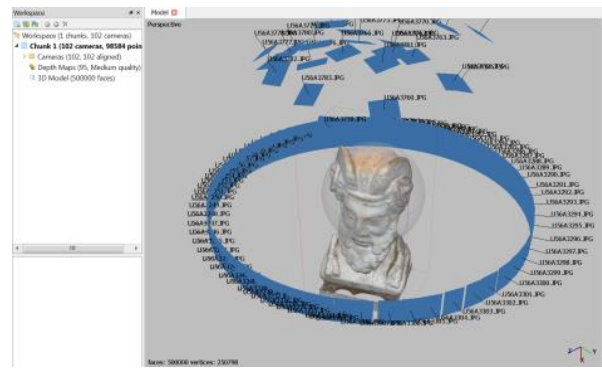

Figure 6. Agisoft PSPro-2 sets alignment

By the use of "rectangular mask" tool, rough masks where used to obtain the alignment at: "Medium accuracy" plus "Generic pre-selection" parameters, not to exclude blurry images (some Top ones). Then again importing created "masks from model" refining it by "intelligent scissors" then the model was generated by setting: Build Geometry, "High quality", "Arbitrary object"; "Smooth geometry" (10GB of RAM at peak). Building Texture command followed, where some top blurred pictures were disabled for a more qualitative result. The meshed textured surface was successfully created with 4.000.000 Triangle faces and 2.001.492 points and the high quality of the results can be appreciated all over the meshed model as in detail e.g. on lateral dimples of the mouth (FIG7). The use of reference points as markers on image base allowed an accurate scaling and successively the local referencing of the model. Furthermore we observe, that an uncontrolled set of images with an variable lightning has to be avoided to get a regular and un-blurry mesh, even the application of this procedure on larger objects like an excavation site with uneven background would not create such problems giving fast, accurate results, as Archaeology asks for.

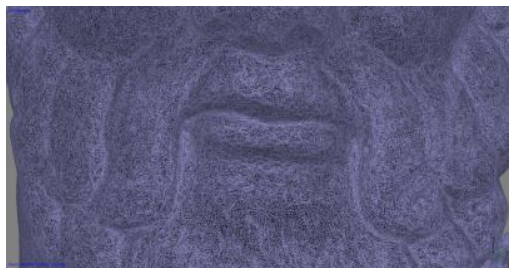

Figure 7. Mesh, depth resolution on mouth details

\section{GIGAPIXEL IMAGES}

\section{THE “PLATE WITH ELEPHANT" FROM CAPENA}

The Cultural Heritage digital imaging activity, definitely one of today's photography branches that best exploits the possibilities offered by the "new" digital technology, firstly applied to provide images free from optical and geometrical distortions, even with a proper restoration of subject colour, and successively to realize, through a series of shots, single high resolution and high quality photos that would otherwise have been impossible to get. "Multi-shot images", series of pictures taken through appropriate shooting techniques, combined with the use of sophisticated software and elaborated workflows are the core of the so-called "Computational Photography", a set of methodologies designed to create a "digital representation" of the subject to provide important information not traceable in individual shots.

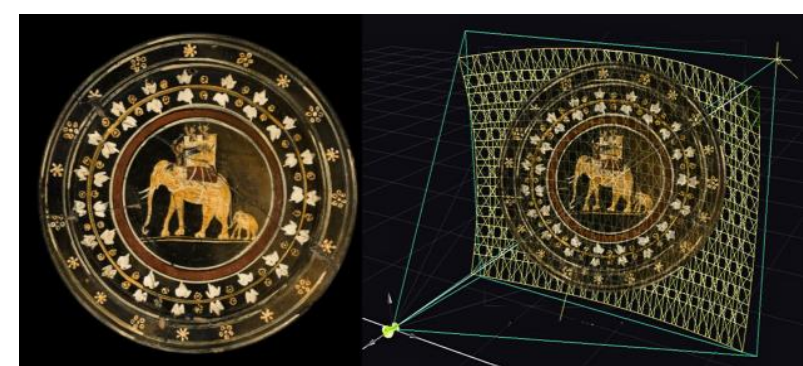

Figure 8. Giga Image and images stitching model

For the so-called "plate with the elephant", we specifically decided to create a GigaPixel image (FIG8), such meaning for the method of gathering multiple shots, partially overlapped of the same subject then joined together (stitching) by the use of dedicated software. The aim was to use a series of 117 shots taken by macrophotography, to obtain an image with such a resolution in pixels that could be greatly zoomed without loss of sharpness in details, thus allowing any accurate study about the state of preservation and of decorative body of the evidence. The end result let the user zoom in, to quickly contextualize every detail in examination, affording an efficient, precise analysis. 


\subsection{The Imaging project and its workflow}

The find is described by Paolo Moreno in the Encyclopaedia of Ancient Art (Treccani, 1965) as a black-painted ceramic plate with over-painted polychromatic decorations, as part of a group of vases called "Pocola". These vases, produced in Lazio region in the first half of the third century B.C., were for votive and/or cult use. This thesis is confirmed by the presence of holes for hanging the pottery on walls and by the fact that the pigments used for the decorations are not very stable. The colours used to decorate the plate were white, brown-yellow and red, fairly dense and vivid, applied in spots onto the black paint.

Furthermore Moreno, on describing the plate, writes that the reproduction of the war-elephant, an Asian breed rather unusual among the oldest in Western world, is the same (except for the baby elephant) as the one engraved on silver Phalerae from Crimea. He thus suggests an earlier common iconography, perhaps Syrian in origin. The plate, kept at Aléria Museum besides most likely referring to the same iconographic model has several points in common with the one we worked on: a votive use and an extremely similar central scene, with the war elephant here followed by a calf.

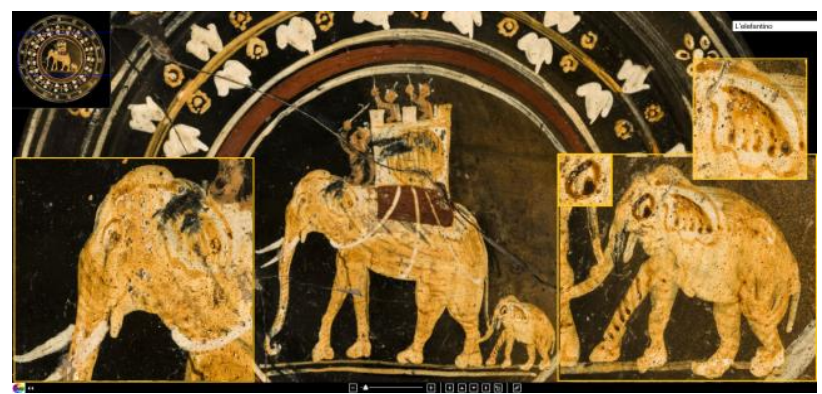

Figure 9 interactive zoom on details

It therefore seemed logical to produce a Giga-image that could help to investigate similarities and differences between the two objects in order to allow the study and an accurate comparison. Once performed even the Aléria plate digital stitching procedure, by the use of an optimized viewer for side-by-side comparison of the two pieces - provided with synchronised pan and zoom functions and with the measuring tool activated - any single person will be able to compare both artefacts in a detailed manner, though hundreds of kilometres far.

The aim of this project is to provide both, the Jerome Carcopino Departmental Museum of Archaeology in Aléria and the Villa Giulia Etruscan National Museum in Rome, with the skill, by virtual connecting the two objects, to better promote them as Cultural Commons.

The photos were taken with a Canon 7D and a SIGMA APO MACRO $150 \mathrm{~mm} \mathrm{f} / 2.8$ that, because of the multiplication factor due to the APS-C type sensor, turns out to be $240 \mathrm{~mm}$ in $35 \mathrm{~mm}$ format. The whole was mounted on a motorized GigaPan Epic PRO head which allows, through micrometric displacements, to create extremely precise shooting grids. Shooting the plate required 13 lines in 9 columns, for a total of 117 shots, which covered a Field of View of $43.41^{\circ} \times 43.35^{\circ}$. The shooting phase involved a set of incandescent lights placed so as to uniformly illuminate the object, even more polarized to avoid reflections. The use of colour references (X-Rite Colour Chart) allowed us to create a colour profile specifically studied for the scene, with which to subsequently de-mosaicing RAW files and carefully apply the white balance right whilst shooting, to achieve a successful colour match between the original and its model. Any single RAW file, once "developed" and optimized with "Lightroom" and "DxO Optics Pro" software to prevent optical and/or geometric distortion, was converted into TIFF 16-bit format and then joined with "Kolor Autopano Giga" sw. The resulting file, PSB $3.63 \mathrm{~Gb}$, with 31.166 x 31.119 pixels, allows a printing size- at 300 dpi resolution - of $264 \times 264 \mathrm{~cm}$.

After the exclusion of the background and the application of a mask for micro-contrast to emphasize fine details, the image was then processed with "Zoomify" one of the most versatile tiling programs.

This software, like other similar ones, "fragments" the image into thousands of tiles, to be used easily both locally then via the web. The end result, actually hosted on the servers of the Soprintendenza per i Beni Archeologici dell'Etruria Meridionale, is a simple-to-use, accurate, and "captivating" product that leads even those who have no particular interest in the matter to carefully observe the subject(FIG9).

\section{3D MODEL IMPORT AND GIS MANAGEMENT}

\subsection{D and 3D GIS Esri Cloud integration of Lidar Survey}

This chapter describes the integration process of the Lidar survey, topographically and GPS oriented, of the archaeological site and of all the sample objects and documents therefore selected into a 2D and 3D GIS environment.

The Esri Gis Software chosen to implement this process has all the capabilities to enable 2D and 3D data, to manage documents and datasets by associating them into a geographic location and to successively allow their integration into the Esri Cloud environment (ArcGIS Online Platform). This has to be taken as an example application inside the actual concept of using the WEB and the CLOUD either as a digital repository to store, promote, compare and share our international Cultural Heritage, but even as a Cloud System, a new paradigm for the provision of computing infrastructures for a wealth of applications classified by the kind of resources they offer: Infrastructure as a Service (IaaS), Platform as a Service (PaaS), and Software as a Service (SaaS), such enhancing or enabling services that we could never before afford.

This project plans to set a specific Cloud Infrastructure for any single organization involved (like the Aléria archaeological site or the Carcopino Museum) and aims by the integration of old historical and new multi resolution datasets resumed with all of these recent and future on site activities, to promote and enhance the sharing of any doings for scientific purposes and also to facilitate the disclosure to tourism and the deriving promotion of the archaeological site.

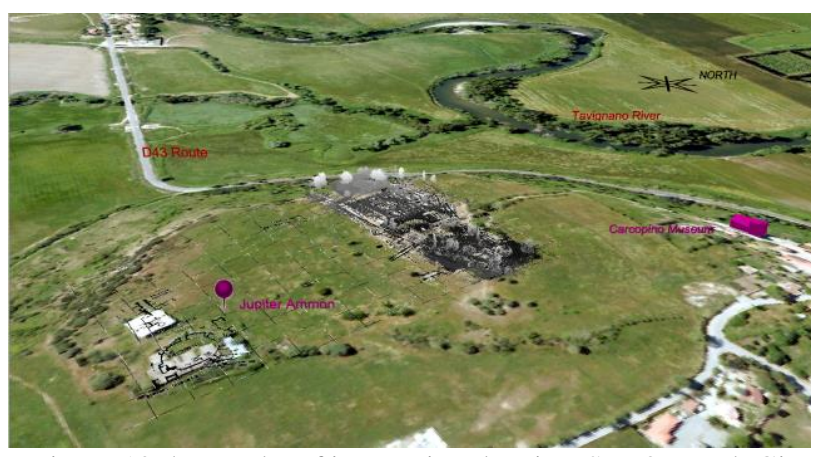

Figure 10 the results of integrating data in ESRI 3D Web Gis

The workflow was performed as follows: the 3D point cloud model topographically and GPS geo-referenced, exported in a PTS file, was imported into the ArcGIS environment after its conversion into .LAS file (a binary format that supports the 
exchange of any 3-dimensional $x, y, z$ tuplet, maintaining information specific to the lidar nature of the data while not being overly complex-ASPRS DEF.). Last ArcGIS Desktop version with $3 D$ Analyst Extension allowed the managing of our Lidar files in LAS format.

Using a specific feature-class called "LAS Dataset", we combined together multiple LAS files (it's often easier to export the 3D point cloud geo-oriented model divided in smaller datasets) displaying them unified in a single layer together with the automatic point filtering, this procedure allowed the easy managing of millions of lidar points in the same dataset within our GIS.

This LAS Dataset constitutes the source from which it's easy to generate different products without replicating any data by the procedure called: "on the fly".

By applying only a "Raster Function", it is possible to generate a DSM (Digital Surface Model) or a DTM (Digital Terrain Model) from Lidar without data replication that would double processing time and costs.

In addition to the Lidar Survey, other information is collected together in the GIS environment:

- Aerial Photos in True Colour 0,5 meter pixel (2007 year),

- SRTM1 (1 arc-second pixel)Digital Terrain Model

- Historical maps of the Archaeological Site

- Historical aerial pictures

- GPS points used to geo-reference the survey used as links

- Digitalized old Pictures and documents(FIG11)

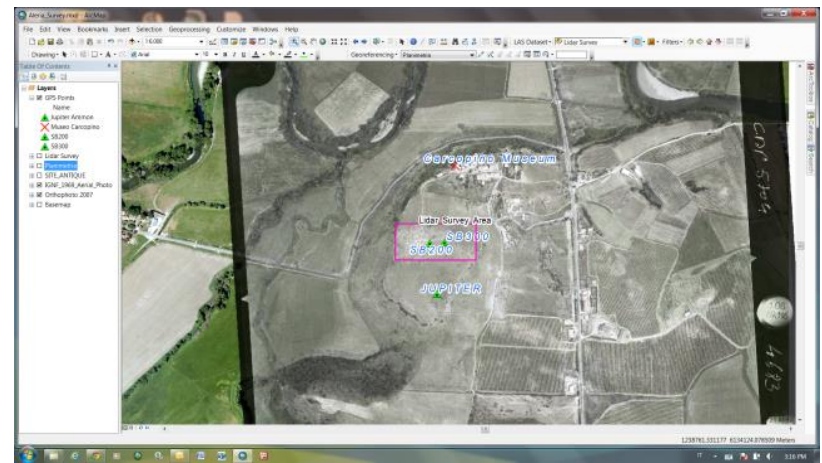

Figure 11. Historical aerial picture into ESRI Web Gis

All of these elements were geo-referenced using the Aerial Photo as a reference. The Coordinate System we used is the same of the Aerial Photo, RGF93 (Réseau Géodésique Français 1993 -EPSG code 2154). Survey LAS file was geo-referenced trough GPS points. The results consist in a completely navigable 2D Application, where it is possible to perform queries on geographic elements or to activate hyperlinks to open documents in any format, as digital or Giga-pixel photos (as we did here) archived in webpages, either filed in the on-site Infrastructure or Platform or in any different one like the one we used (25 GB free space) provided to SAPIENZA by Google.

By the ArcScene application (a 3D Analyst extension).the Lidar survey was integrated in a 3D scene ,by a composition of aerial photos of historical maps and photos draped over SRTM1 DTM, lastly even the lidar survey was draped over the aerial photo with good results.

A ground component of Lidar was extracted and used to correct the Digital Terrain Model derived from SRTM. This process increased the precision of the DTM used as "Base Height" on which to drape layers and to give a 3D effect to scenery. SRTM base Data had in fact a too low resolution with respect to that of Maps Data and Lidar Survey.

In both applications it is possible to use ArcGIS Online basemaps content to change cartographic background for any different use or context.

In the 3D Scene it has been possible to create the 3D model of the Museum building, with a synthesis as we did or a more detailed one, even importing it and stitching pictures on it or, a useful base to perform powerful 3D Analyses (Spatial analysis and Visibility analysis).(FIG10)

After the creation of 2D and 3D maps both were shared in the ArCGIS Online environment (the Esri Cloud Platform environment), using ArcMap and ArcScene applications.

This generated three types of contents:

- 2D navigable content usable of other ArcGIS Clients

- Preconfigured Web Application hosted by Cloud

- 3D Web scene derived by ArcScene (a new functionality of 10.2 ArcGIS version available from 15 July 2013)

This content, just created and loaded In ArcGIS Online, it is shareable as we needed even with all users or with specific ones or defined Groups, the association of specific tags to it makes more simple to retrieve it with the search functionality.

This last demonstration and collection of very up-to-date procedures squares the circle of our project for the international collaboration of know-how's, joined together to the worldwide Cultural Heritage preservation, optimization and sharing. This last looks like the end, but it's just the beginning.

\section{Bibliography:}

-Arrighi, J.M., Jehasse, O., Histoire de la Corse et des Corses, Perrin, 2008

-Carandini, A., Storie dalla terra, Einaudi 2000

-Carpiceci, M., Fotografia Digitale e Architettura, Aracne, 2012 -De Luca, L., La fotomodellazione architettonica, Flaccovio, 2011

-Guidi, G. et Al. Acquisizione 3D e modellazione poligonale, Mc Graw Hill, 2010

-Harris, E.C., Principi di Stratigrafia Archeologica, Carocci, 2009

-Manacorda, D., Lezioni di Archeologia, Laterza 2008

-Archeologia e Calcolatori, ${ }^{\circ} 21$, all'Insegna del Giglio, 2010

-Panella, C. - Gabrielli, R. - Giorgi, C., Le "Terme di Elagabalo" sul Palatino: sperimentazione di un metodo fotogrammetrico 3D applicato allo scavo archeologico

In Archeologia e Calcolatori n. XXII - 2011 pp. 243-260

-Alby, E., Smigiel, E., Assali, P., 2011a. Valorisation numérique du théâtre Gallo-romain de Mandeure. Revue Française de Photogrammétrie et Télédétection $n^{\circ} 196, \mathrm{n}^{\circ} 196$, pp. 2-9.

-Remondino, F., Rizzi, A., 2010. Reality-based 3D documentation of natural and cultural heritage sites: techniques, problems, and examples, Applied Geomatics, 2(3), pp. 85-100

-Pomaska, G., 2008. The Impact of GPS Tagging on Image Based Documentation and 3D Reconstruction of Cultural Assets. Conference on Virtual Systems and Multimedia Dedicated to Cultural Heritage, Limassol, Cyprus

-Boi V., Cordone C., Lamonaca F., Picciola S., Stacca M. 2011, Il SITAR e la modellazione dei dati tridimensionali. Il caso dell'Esquilino e dei Colombari di Vigna in Serlorenzi 2011a, 227-244.

-Arrighetti, A., Cavalieri, M., Il rilievo fotogrammetrico per nuvole di punti RGB della 'sala triabsidata' del sito archeologico di Aiano-Torraccia di Chiusi (SI), Archeologia e Calcolatori n. XXIII - 2012 pp. 121-134 